\title{
Fibonacci collocation method with a residual error function to solve linear Volterra integro differential equations
}

\author{
Ayse Kurt Bahsi and Salih Yalcinbas \\ Department of Mathematics, Celal Bayar University, Manisa, Turkey
}

Received: 5 October 2015, Revised: 20 November 2015, Accepted: 7 December 2015

Published online: 1 January 2016.

\begin{abstract}
In this paper, a new collocation method based on the Fibonacci polynomials is introduced to solve the high-order linear Volterraintegro-differential equations under the conditions. Numerical examples are included to demonstrate the applicability and validity of the proposed method and comparisons are made with the existing results. In addition, an error estimation based on the residual functions is presented for this method. The approximate solutions are improved by using this error estimation.
\end{abstract}

Keywords: Volterra integral and integro-differential equations, Fibonacci polynomials, residual error analysis, collocation points, matrix method.

\section{Introduction}

In recent years, the integral and integro-differential equations have a major role in engineering, mechanics, physics, chemistry, astronomy, biology, economics, potential theory, electrostatics, (Kadalbajoo \& Sharma 2002, Elmer \& Van Vleck 2002, Maleknejad \& Mahmoudi 2004, Cao \& Wang 2004, Kadalbajoo \& Sharma 2004). A physical event can be modeled by these equations. Since few of these equations cannot be solved explicitly, it is necessary to resort to the numerical techniques. Many authors have worked on numerical methods such as Adomian decomposition method (Wazwaz 2010), the CAS wavelet method (Danfu \& Xufeng, 2007), the Galerkin method (Brunner et al, 2009), the Tau method (Pour-Mahmoud, 2005), the finite difference method (Zhao \& Corless, 2006), the Haar function method (Maleknejad \& Mirzaee, 2006), the sine-cosine wavelet methods (Kajani et al, 2007) for solutions of these equations.

Since the beginning of 1994, for solving the linear and nonlinear Volterra integral and Volterraintegro-differential, Fredholm-Volterraintegro-differential equation matrix methods have also been used by Sezer et al. (Sezer 1994, Yalcinbas \& Sezer 2000, Yalcinbas 2002, Sorkun \& Yalcinbas 2010, Yuzbasi et al 2011) Also, the Fibonacci matrix method has been used to find the approximate solutions of differential, integral and integro-differential equations, differential- difference equations and Fredholmintegro differential-difference equations (Kurt 2012, Kurt et al 2013a, 2013b).

In this study, we consider the approximate solution of the $\mathrm{m} t h$-order Volterraintegro differential equations,

$$
\sum_{k=0}^{m} P_{k}(x) y^{(k)}(x)=g(x)+\lambda \int_{a}^{x} K(x, t) y(t) d t, 0 \leq a \leq x, t \leq b
$$


under the conditions

$$
\sum_{k=0}^{m-1}\left[a_{j k} y^{(k)}(a)+b_{j k} y^{(k)}(b)\right]=\lambda_{j}, j=1,2,3, \ldots, m
$$

where $P_{k}(x), g(x)$, and $K(x, t)$ are functions defined on $a \leq x, t \leq b ; a_{j k}, b_{j k}, \lambda$ and $\lambda_{j}$ are suitable constants. Our aim is to obtain an approximate solution of (1) expressed in the truncated Fibonacci series form

$$
y(x)=\sum_{n=1}^{N} a_{n} F_{n}(x)
$$

$a_{n}, n=1,2,3, \ldots, N$ are the unknown Fibonacci coefficients. Here $N$ is chosen any positive integer such that $N \geq m$ and $F_{n}(x), n=1,2,3, \ldots, N$ are the Fibonacci polynomials defined by

$$
\begin{gathered}
F_{n}(x)=\sum_{j=0}^{[(n-1) / 2]}\left(\begin{array}{c}
n-j-1 \\
j
\end{array}\right) x^{n-2 j-1}, \\
{[(n-1) / 2]=\left\{\begin{array}{l}
(n-2) / 2, n \text { even } \\
(n-1) / 2, n \text { odd } .
\end{array}\right.}
\end{gathered}
$$

\section{Fundamental matrix relations}

We first write the Fibonacci polynomials $F_{n}(x)$ in the matrix form as follows

$$
F^{T}(x)=C X^{T}(x) \Leftrightarrow F(x)=X(x) C^{T}
$$

where $F(x)=\left[F_{1}(x) F_{2}(x) \ldots F_{N}(x)\right]$,

$$
X(x)=\left[1 x \ldots x^{N-1}\right]
$$

if $N$ is even,

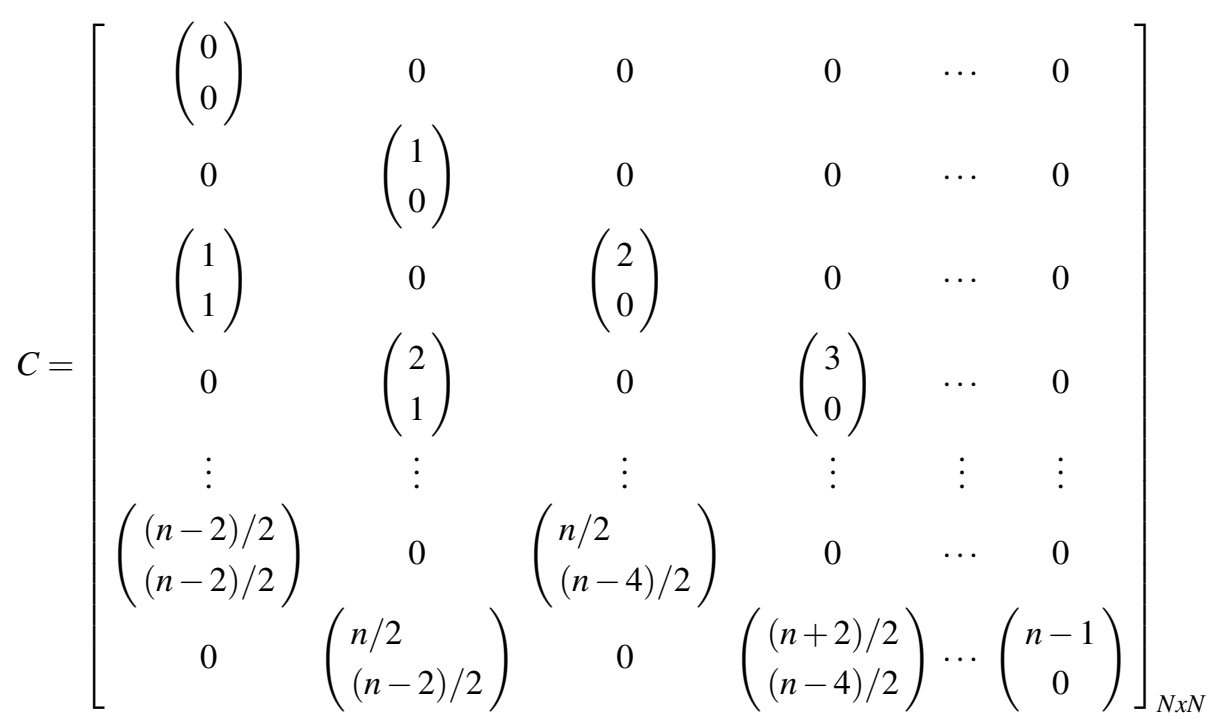


if $N$ is odd,

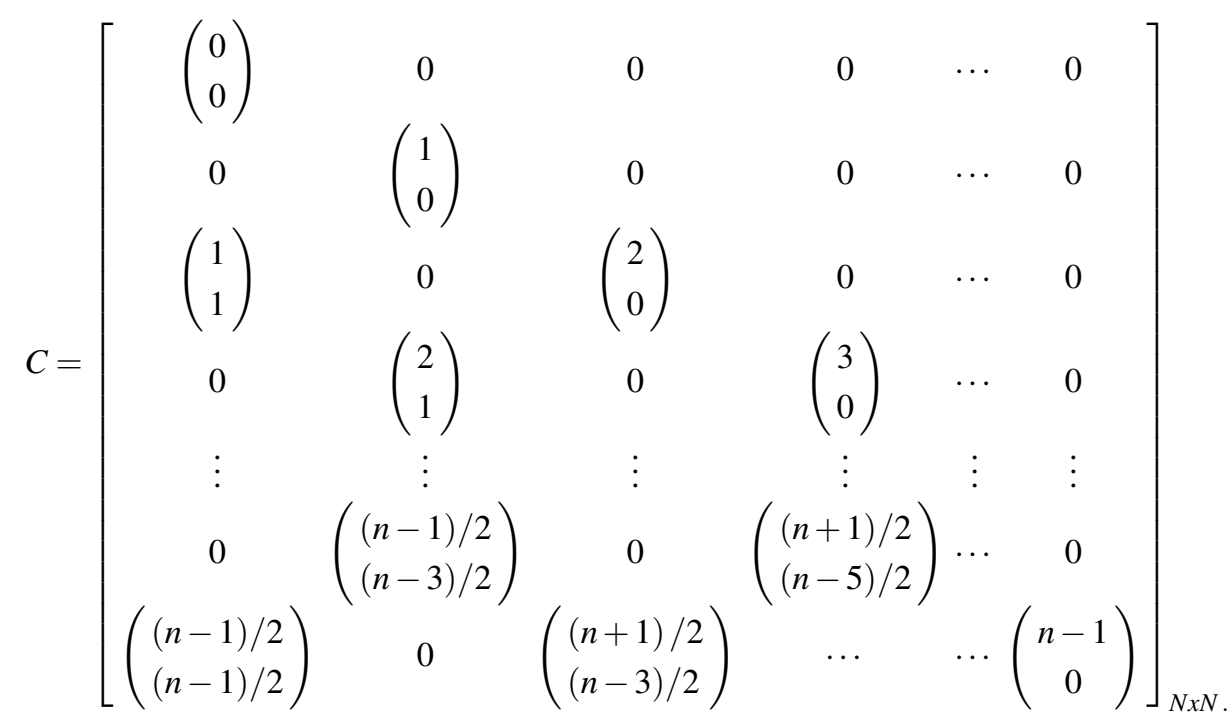

Let us show Eq.(1) in the form

$$
P(x)=g(x)+\lambda I(x)
$$

where

$$
P(x)=\sum_{k=0}^{m} P_{k}(x) y^{(k)}(x) .
$$

\section{Matrix relations for the differential part $\mathbf{P}(X)$}

We consider the solution $y(x)$ and its kth derivate $y^{(k)}(x)$ in the matrix form

$$
\begin{gathered}
y(x)=F(x) A, A=\left[\begin{array}{llll}
a_{1} & a_{2} & \cdots & a_{N}
\end{array}\right]^{T} \\
y^{(k)}(x)=F^{(k)}(x) A .
\end{gathered}
$$

Then, from relations (4) and (7), we can obtain the following matrix form

$$
y(x)=X(x) C^{T} A
$$

Similar to Eq. (8), from relations (4), (7) and (8), we can find $y^{(k)}(x)$ matrix form as

$$
y^{(k)}(x)=X^{(k)}(x) C^{T} A .
$$

To find the matrix $X^{(k)}(x)$ in terms of the matrix $X(x)$, we can use the following relation

$$
\begin{gathered}
X^{(0)}(x)=X(x) \\
X^{(1)}(x)=X(x) B^{T}
\end{gathered}
$$




$$
\begin{gathered}
X^{(2)}(x)=X^{(1)}(x) B^{T}=\left(X(x) B^{T}\right) B^{T}=X(x)\left(B^{T}\right)^{2} \\
\vdots \\
X^{(k)}(x)=X^{(k-1)}(x) B^{T}=X(x)\left(B^{T}\right)^{k}
\end{gathered}
$$

where

$$
B^{T}=\left[\begin{array}{ccccccc}
0 & 1 & 0 & \cdots & 0 & 0 & 0 \\
0 & 0 & 2 & \cdots & 0 & 0 & 0 \\
0 & 0 & 0 & \cdots & 0 & 0 & 0 \\
\vdots & \vdots & \vdots & & \vdots & & \vdots \\
0 & 0 & 0 & \cdots & 0 & 0 & N-1 \\
0 & 0 & 0 & \cdots & 0 & 0 & 0
\end{array}\right] .
$$

By using the relations (9)-(11) we have the recurrence relations

$$
y^{(k)}(x)=X(x)\left(B^{T}\right)^{k} C^{T} A .
$$

Subsequently, by substituting the matrix form (12) into Eq.(6), we obtain the matrix relations

$$
P(x)=\sum_{k=0}^{m} P_{k}(x) X(x)\left(B^{T}\right)^{k} C^{T} A .
$$

\section{Matrix relations for the integral part $I(X)$}

Let us find the matrix relation for the Volterra integral part $I(x)$ in Eq.(6). The kernel function $K(x, t)$ can be shown by the truncated Fibonacci series,

$$
K(x, t)=\sum_{m=0}^{N} \sum_{n=0}^{N} k_{m n}^{f} F_{m}(x) F_{n}(t)
$$

and the truncated Taylor series,

$$
K(x, t)=\sum_{m=0}^{N} \sum_{n=0}^{N} k_{m n}^{t} x^{m} t^{n}
$$

where

$$
k_{m n}^{t}=\frac{1}{m ! n !} \frac{\partial^{m+n} K(0,0)}{\partial x^{m} \partial t^{n}} ; m, n=0,1, \ldots N-1 .
$$

The expressions (14)-(15) can be put matrix forms as

$$
K(x, t)=F(x) K_{F} F^{T}(t), K_{F}=\left[k_{m n}^{f}\right]
$$

and

$$
K(x, t)=X(x) K_{t} X^{T}(t), K_{t}=\left[k_{m n}^{t}\right] .
$$

From (16)-(17) we can obtain

$$
X(x) K_{t} X^{T}(t)=F(x) K_{F} F^{T}(t) \Rightarrow X(x) K_{t} X^{T}(t)=X(x) C^{T} K_{F} C X^{T}(t)
$$


thus

$$
K_{t}=C^{T} K_{F} C \Rightarrow K_{F}=\left(C^{T}\right)^{-1} K_{t} C^{-1} .
$$

By substituting the matrix forms (7) and (17) into the integral part $I(x)$ in (5). So we can have the matrix relation as follows

$$
[I(x)]=\int_{a}^{x} F(x) K_{F} F^{T}(t) F(t) A d t=F(x) K_{F} Q(x) A
$$

so that

$$
[Q(x)]=\int_{a}^{x} F^{T}(t) F(t) d t=\int_{a}^{x} C X^{T}(t) X(t) C^{T} d t=C H(x) C^{T}
$$

where $H(x)=\left[h_{i j}(x)\right]=\int_{a}^{x} X^{T}(t) X(t) d t$,

$$
h_{i j}(x)=\frac{x^{i+j+1}-a^{i+j+1}}{i+j+1} i, j=1,2, \ldots, N .
$$

If we subsitute the matrix relation (4) into (19), we have the matrix form

$$
[I(x)]=X(x) M H(x) C^{T} A
$$

where

$$
M=C^{T} K_{F} C .
$$

\section{Matrix relations for the conditions}

The corresponding matrix form for the conditions (2) can be shown, by means of (12), as

$$
\sum_{k=0}^{m-1}\left[a_{j k} X(a)+b_{j k} X(b)\right]\left(B^{T}\right)^{k} C^{T} A=\lambda_{j}, j=1,2, \ldots, m .
$$

\section{Method of solution}

We can construct the fundamental matrix equation corresponding for Eq.(1). For this aim, we substitute the matrix relations (13) and (20) into Eq. (5). So we obtain the matrix equation

$$
\sum_{k=0}^{m} P_{k}(x) X(x)\left(B^{T}\right)^{k} C^{T} A=g(x)+\lambda X(x) M H(x) C^{T} A .
$$

By using in Eq. (22) the collocation points $x_{i}$ defined by $x_{i}=a+\left(\frac{b-a}{N-1}\right)(i-1), i=1,2, \ldots, N$. The system of the matrix equations is obtained

$$
\sum_{k=0}^{m} P_{k}\left(x_{i}\right) X\left(x_{i}\right) C^{T}\left(B^{T}\right)^{k} A=g\left(x_{i}\right)+\lambda X\left(x_{i}\right) M H\left(x_{i}\right) C^{T} A .
$$

or shortly the fundamental matrix equation becomes

$$
\left\{\sum_{k=0}^{m} P_{k} X\left(B^{T}\right)^{k} C^{T}-\lambda \bar{X} \bar{M} \bar{H} \bar{C}\right\} A=G
$$

where 


$$
\begin{aligned}
& P_{k}=\left[\begin{array}{cccc}
P_{k}\left(x_{1}\right) & 0 & \cdots & 0 \\
0 & P_{k}\left(x_{2}\right) & \cdots & 0 \\
\vdots & \vdots & \ddots & 0 \\
0 & 0 & \cdots & P_{k}\left(x_{N}\right)
\end{array}\right], \quad G=\left[\begin{array}{c}
g\left(x_{1}\right) \\
g\left(x_{2}\right) \\
\vdots \\
g\left(x_{N}\right)
\end{array}\right], \quad X=\left[\begin{array}{c}
X\left(x_{1}\right) \\
X\left(x_{2}\right) \\
\vdots \\
X\left(x_{N}\right)
\end{array}\right]=\left[\begin{array}{cccc}
1 & x_{1} & \cdots & x_{1}^{N-1} \\
1 & x_{2} & \cdots & x_{2}^{N-1} \\
\vdots & \vdots & \ddots & \vdots \\
1 & x_{N} & \cdots & x_{N}^{N-1}
\end{array}\right], \\
& \bar{X}=\left[\begin{array}{cccc}
X\left(x_{1}\right) & 0 & \cdots & 0 \\
0 & X\left(x_{2}\right) & \cdots & 0 \\
\vdots & \vdots & \vdots & \vdots \\
0 & 0 & \cdots & X\left(x_{N}\right)
\end{array}\right] \\
& \bar{M}=\left[\begin{array}{cccc}
M & 0 & \cdots & 0 \\
0 & M & \cdots & 0 \\
\vdots & \vdots & \vdots & \vdots \\
0 & 0 & \cdots & M
\end{array}\right], \bar{C}=\left[\begin{array}{c}
C^{T} \\
C^{T} \\
\vdots \\
C^{T}
\end{array}\right], \quad \bar{H}=\left[\begin{array}{cccc}
H_{v}\left(x_{1}\right) & 0 & \cdots & 0 \\
0 & H_{v}\left(x_{2}\right) & \cdots & 0 \\
\vdots & \vdots & \vdots & \vdots \\
0 & 0 & \cdots & H_{v}\left(x_{N}\right)
\end{array}\right], A=\left[\begin{array}{c}
a_{1} \\
a_{2} \\
\vdots \\
a_{N}
\end{array}\right] .
\end{aligned}
$$

Therefore, the fundamental matrix equation (23) corresponding for Eq. (1) can be written as

$$
W A=G
$$

where

$$
W=\sum_{k=0}^{m} P_{k} X\left(B^{T}\right)^{k} C^{T}-\lambda \bar{X} \bar{M} \bar{H} \bar{C}
$$

Eq. (24) corresponds to a system of $N$ linear algebraic equations with unknown Fibonacci coefficients $a_{1}, a_{2}, \ldots, a_{N}$. Further, we can express the matrix form (21) conditions

$$
U_{j} A=\left[\lambda_{j}\right]
$$

where

$$
U_{j}=\sum_{k=0}^{m-1}\left[a_{j k} X(a)+b_{j k} X(b)\right]\left(B^{T}\right)^{k} C^{T}=\left[\begin{array}{lllll}
u_{j 1} & u_{j 2} & u_{j 3} & \cdots & u_{j N}
\end{array}\right] .
$$

To obtain the solution of Eq. (1) under the conditions (2), by replacing the row matrices (25) by the last $m$ rows of the matrices (24) (or any $m$ rows of the matrix (24)), we have the new augmented matrix,

$$
[\tilde{W} ; \tilde{G}]=\left[\begin{array}{cccccc}
w_{11} & w_{12} & \cdots & w_{1 N} & ; & g\left(x_{1}\right) \\
w_{21} & w_{22} & \cdots & w_{2 N} & ; & g\left(x_{2}\right) \\
\vdots & \vdots & \vdots & \vdots & ; & \vdots \\
w_{(N-m) 1} & w_{(N-m) 2} & \cdots & w_{(N-m) N} & ; & g\left(x_{N-m}\right) \\
u_{11} & u_{12} & \cdots & u_{1 N} & ; & \lambda_{1} \\
u_{21} & u_{22} & \cdots & u_{2 N} & ; & \lambda_{2} \\
\vdots & \vdots & \vdots & \vdots & ; & \vdots \\
u_{m 1} & u_{m 2} & \cdots & u_{m N} & ; & \lambda_{m}
\end{array}\right] .
$$

If $\operatorname{rank} \tilde{W}=\operatorname{rank}[\tilde{W} ; \tilde{G}]=N$, then we can write

$$
A=(\tilde{W})^{-1} \tilde{G}
$$


And so, the matrix $A$ (thereby the coefficients $a_{1}, a_{2}, \ldots, a_{N}$ ) is uniquely determined. Therefore, Eq. (1) with conditions (2) has a unique solution which is given by Fibonacci series solution (3). On the other hand, when $|\tilde{W}|=0$, that is if rank $\tilde{W}=\operatorname{rank}[\tilde{W} ; \tilde{G}] \leq N$, we can find a particular solution. Otherwise if $\operatorname{rank} \tilde{W} \neq \operatorname{rank}[\tilde{W} ; \tilde{G}] \leq N$, then there is no solution.

\section{Residual function and error estimation}

In this section, we will give an efficient error estimation for the Fibonacci polynomial approximation and also a technique to obtain the corrected solution of the problem (1) and (2) by using the residual correction method (Oliveira 1980, Çelik 2006) For our aim, we define the residual function for the present method as

$$
R_{N}(x)=\sum_{k=0}^{m} P_{k}(x) y_{N}{ }^{(k)}(x)-\lambda \int_{a}^{x} K(x, t) y_{N}(t) d t-g(x)
$$

where $y_{N}(x)$ is the approximate solution of the problem (1)-(2). Note that, $y_{N}(x)$ satisfies the following problem

$$
\begin{aligned}
& \sum_{k=0}^{m} P_{k}(x) y_{N}{ }^{(k)}(x)-\lambda \int_{a}^{x} K(x, t) y_{N}(t) d t=g(x)+R_{N}(x) \\
& \sum_{k=0}^{m-1}\left[a_{j k} y_{N}^{(k)}(0)+b_{j k} y_{N}^{(k)}(b)\right]=\lambda_{j}, j=1,2,3, \ldots, m .
\end{aligned}
$$

In addition, the error function $e_{N}(x)$ can be defined as

$$
e_{N}(x)=y(x)-y_{N}(x)
$$

where $y(x)$ is the exact solution of the problem (1) - (2). Substituting (29) into (1) and (2) and using (27) and (28), we have the error differential equation with the homogenous conditions

$$
\begin{aligned}
& \sum_{k=0}^{m} P_{k}(x) e_{N}{ }^{(k)}(x)-\lambda \int_{a}^{x} K(x, t) e_{N}(t) d t=-R_{N}(x) \\
& \sum_{k=0}^{m-1}\left[a_{j k} e_{N}^{(k)}(0)+b_{j k} e_{N}^{(k)}(b)\right]=0, j=1,2,3, \ldots, m .
\end{aligned}
$$

Solving the problem (30) in the same way as in Section 3, we get the approximation $e_{N, M}(x)$ to $e_{N}(x), M>N$ which is the error function based on the residual function.

Subsequently, by means of the Fibonacci polynomials $y_{N}(x)$ and $e_{N, M}(x)$, we obtain the corrected solution

$$
y_{N, M}(x)=y_{N}(x)+e_{N, M}(x) .
$$

Also, we construct the Fibonacci error function $e_{N}(x)=y(x)-y_{N}(x)$, and the corrected Fibonacci error function

$$
E_{N, M}(x)=e_{N}(x)-e_{N, M}(x)
$$




\section{Numerical examples}

In this section, several examples are given to illustrate the applicability of the Fibonacci matrix method and all of them are performed on the computer. In examples, the terms $\left|e_{N}(x)\right|$ and $\left|E_{N, M}(x)\right|$ respectively, represent the absolute error function and the absolute error function of corrected Fibonacci polynomial solution.

Example 1. (Kurt 2012) Let us consider the linear Volterraintegro-differential equation given by

$$
\begin{aligned}
& \left(x^{3}-1\right) y^{\prime \prime}(x)+2 x y^{\prime}(x)-y(x)=-2 x^{4}+5 x^{3}+15 x^{2}+3 x-7+6 \int_{0}^{x}(x-t) y(t) d t \\
& y(0)=-1, y^{\prime}(-1)=-5,0 \leq x, t \leq 2
\end{aligned}
$$

with the exact solution

$$
y(x)=4 x^{2}+3 x-1
$$

The approximate solution $y(x)$ by the truncated Fibonacci series

$$
y(x)=\sum_{n=1}^{N} a_{n} F_{n}(x)
$$

where

$$
N=4, p_{0}(x)=-1, p_{1}(x)=2 x, p_{2}(x)=x^{3}-1, g(x)=-2 x^{4}+5 x^{3}+15 x^{2}+3 x-7, \lambda=6, K(x, t)=x-t
$$

The collocation points for $N=3$ are computed as

$$
\left\{x_{1}=0, x_{2}=\frac{2}{3}, x_{3}=\frac{4}{3}, x_{4}=2\right\}
$$

and from Eq.(22), the fundamental matrix equation of the problem is

$$
\left\{P_{0} X C^{T}+P_{1} X B^{T} C^{T}+P_{2} X\left(B^{T}\right)^{2} C^{T}-\lambda \bar{X} \bar{M} \bar{H} \bar{C}\right\} A=G
$$

The augmented matrix for this fundamental matrix equations is

$$
[W ; G]=\left[\begin{array}{cccccc}
-1 & 0 & -3 & 0 & ; & -7 \\
-\frac{7}{3} & \frac{10}{27} & -\frac{203}{81} & -\frac{256}{405} & ; & \frac{223}{81} \\
-\frac{19}{3} & -\frac{28}{27} & \frac{13}{81} & \frac{2493}{128} & ; & \frac{2365}{81} \\
-13 & -6 & 5 & \frac{512}{5} & ; & 67
\end{array}\right]
$$

From Eq. (24), the matrix form for the conditions are $U_{j} A=\left[\lambda_{j}\right]$ or $\left[U_{j} ; \lambda_{j}\right] ; j=0,1$ or clearly

$$
\begin{aligned}
& {\left[U_{0} ; \lambda_{0}\right]=\left[\begin{array}{lllll}
1 & 0 & 1 & 0 & 0
\end{array}-1\right]}
\end{aligned}
$$

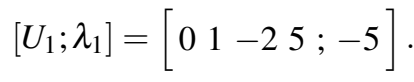


From Eq. (25), we can obtain the new augmented matrix based on the condition as follows

$$
[\tilde{W} ; \tilde{G}]=\left[\begin{array}{ccccc}
-1 & 0 & -3 & 0 & ;-7 \\
-\frac{7}{3} & -\frac{10}{27} & -\frac{203}{81} & -\frac{256}{405} ; \frac{223}{81} \\
1 & 0 & 1 & 0 & ;-1 \\
0 & 1 & -2 & 5 & ;-5
\end{array}\right]
$$

When we solve this system, we find the Fibonacci coefficients matrix

$$
A=\left[\begin{array}{llll}
-5 & 3 & 4 & 0
\end{array}\right]^{T}
$$

Hence, by substituting the Fibonacci coefficients matrix into Eq. (7), we have the approximate solution $y(x)=4 x^{2}+3 x-1$, which is the exact solution.

Example 2. (Yalcinbas \& Sezer 2000) Consider the linear Volterraintegro-differential equation

$$
y^{\prime}(x)=1-\int_{0}^{x} y(t) d t, 0 \leq x, t \leq 1
$$

with $y(0)=0$ and the exact solution $y(x)=\sin (x)$. From Eq. (23), the fundamental matrix equation is

$$
\left\{P_{1} X B^{T} C^{T}-\lambda \bar{X} \bar{M} \bar{H} \bar{C}\right\} A=G
$$

where $N=6, p_{1}(x)=1, g(x)=1, \lambda=-1, K(x, t)=1$. Thus, we obtain the solution of the problem for $N=6$,

$$
\begin{aligned}
& y_{6}(x)=(0.762454157653902 e-2) x^{5}+(0.815190187782200) x^{4} \\
& -(0.167011007897479) x^{3}+(0.519273963677236 e-4) x^{2}+x .
\end{aligned}
$$

In order to estimate the errors for $N=6$ we consider the following error problem of (30)

$$
\begin{aligned}
& e_{6}{ }^{\prime}(x)-1+\int_{0}^{x} e_{6}(t) d t=-R_{6}(\mathrm{x}) \\
& e_{6}(0)=0 .
\end{aligned}
$$

Here the residual function is

$$
R(x)=e_{6}^{\prime}(x)-1+\int_{0}^{x} e_{6}(t) d t .
$$

By solving the error problem (33) for $M=8$ introduced in Section 4, the estimated error function approximation $e_{6,8}(x)$ is obtained

$$
\begin{aligned}
& e_{6,8}(x)=-(0.179550071001374 e-3) x^{7}-(0.346191640591268 e-4) x^{6}+(0.736607283607877 e-3) x^{5} \\
& -(0.826804594556656 e-3) x^{4}+(0.346824082083329 e-3) x^{3}-(0.521485598362318 e-4) x^{2} \\
& +(0.299239799605999 e-14) x+(0.216840434497101 e-18)
\end{aligned}
$$

Hence, we obtain the corrected exponential solution

$$
e_{6,8}(x)=y_{6}(x)+e_{6,8}(x)
$$


It is seen from Table 1, that the results obtained by the present method is very superior to that obtained by the Taylor method (Yalcinbas \& Sezer, 2000). We compare the absolute error $\left|e_{6}\left(x_{i}\right)\right|$ with the corrected absolute error $\left|E_{6,8}\left(x_{i}\right)\right|$ in Table 2 which shows the numerical results of the absolute errors and the corrected absolute errors for $N=8, M=9$. In addition, Figure 1 illustrates a comparison of the absolute error with the corrected absolute error. It indicates the significant decrease in the absolute error owing to the error correction by the residual function

\begin{tabular}{|c|c|c|c|c|}
\hline & Exact solution & Taylor method [14] & \multicolumn{2}{|c|}{ Present method } \\
\cline { 2 - 5 } & $N=5$ & $N=6, M=7$ & & $y_{6,8}\left(x_{i}\right)$ \\
\hline$x_{i}$ & $y\left(x_{i}\right)=\sin \left(x_{i}\right)$ & $y_{5}\left(x_{i}\right)$ & $y_{6}\left(x_{i}\right)$ & 0.00000000 \\
\hline 0.0 & 0.00000000 & 0.00000000 & 0.00000000 & 0.19866933 \\
\hline 0.2 & 0.19866933 & 0.19866933 & 0.19866973 & 0.38941834 \\
\hline 0.4 & 0.38941834 & 0.38941866 & 0.38941854 & 0.56464247 \\
\hline 0.6 & 0.56464247 & 0.56464800 & 0.56464284 & 0.71735609 \\
\hline 0.8 & 0.71735609 & 0.71739733 & 0.7173559 & 0 \\
\hline
\end{tabular}

Table 1: Comparison of the solutions of Example 5.2.

\begin{tabular}{|c|c|c|}
\hline \multirow{2}{*}{$x_{i}$} & Absolute error & \multicolumn{2}{|c|}{ Corrected absolute error } \\
\cline { 2 - 3 } & $\left|e_{6}\left(x_{i}\right)\right|$ & $\left|E_{6,8}\left(x_{i}\right)\right|$ \\
\hline 0.0 & 0 & 0 \\
\hline 0.2 & $4.02395202 \mathrm{e}-7$ & $6.41420 \mathrm{e}-10$ \\
\hline 0.4 & $2.05743865 \mathrm{e}-7$ & $5.98424 \mathrm{e}-10$ \\
\hline 0.6 & $3.75763114 \mathrm{e}-7$ & $4.54363 \mathrm{e}-10$ \\
\hline 0.8 & $1.81724658 \mathrm{e}-7$ & $1.45354 \mathrm{e}-10$ \\
\hline 1.0 & $9.66645529 \mathrm{e}-6$ & $2.45684 \mathrm{e}-08$ \\
\hline
\end{tabular}

Table 2: Numerical results of the error functions of Example 5.2 for $N=8, M=9$.

Example 3. (Hosseini \& Shahmorad 2002) With exact solution $y(x)=e^{x^{2}}$ consider the linear Volterraintegro-differential equation

$$
\begin{aligned}
& y^{\prime}(x)+y(x)=1+2 x+\int_{0}^{x} x(1+2 x) e^{t(x-t)} y(t) d t, 0 \leq x, t \leq 1, \\
& y(0)=1
\end{aligned}
$$

From Eq. (23), the fundamental matrix equation of the problem is

$$
\left\{P_{0} X C^{T}+P_{1} X B^{T} C^{T}-\lambda \bar{X} \bar{M} \bar{H} \bar{C}\right\} A=G
$$

where $p_{0}(x)=1, p_{1}(x)=1, g(x)=1+2 x, \lambda=1, K(x, t)=x(1+2 x) e^{t(x-t)}$.

The absolute errors of the solutions obtained by the other methods (Hosseini \& Shahmorad 2002, Makroglou 1980)are compared with the absolute error of the solutions obtained by presented method for $N=11$ in the Table 3 . In Table 4 and Figure 3, we compare the corrected absolute error $\left|E_{11.12}\left(x_{i}\right)\right|$ with the corrected absolute errors $\left|E_{11.14}\left(x_{i}\right)\right|$ and $\left|E_{11.17}\left(x_{i}\right)\right|$. It reveals that the residual function will give more accurate results with the smaller corrected errors. 


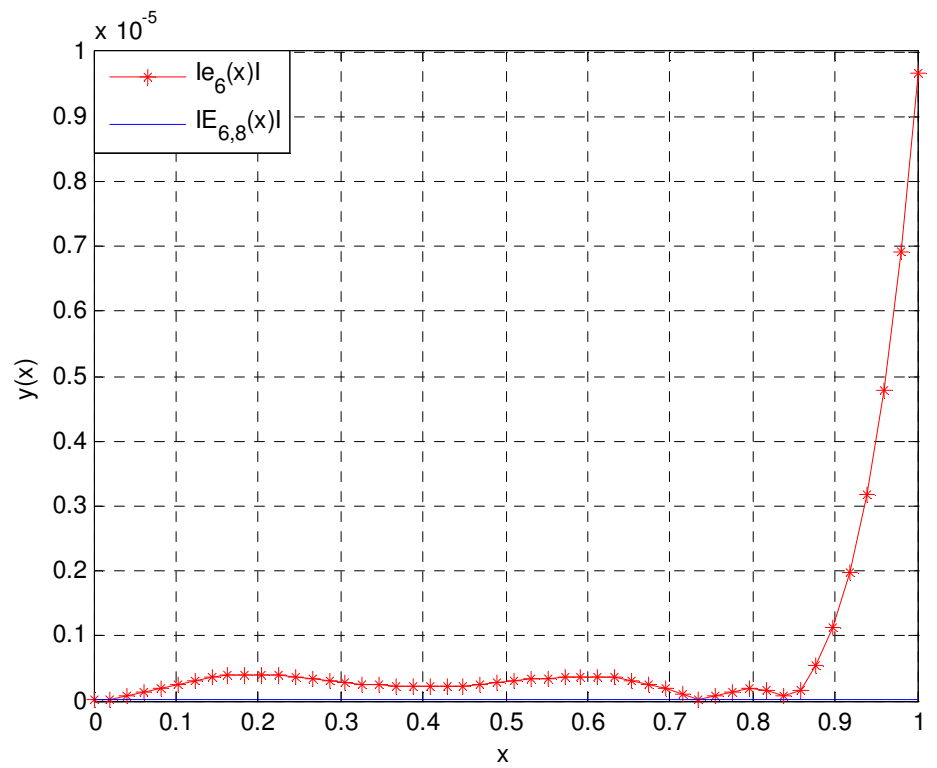

Fig. 1: Comparison of the absolute error with the corrected absolute error for Eq.(32).

\begin{tabular}{|c|c|c|c|c|}
\hline \multirow{2}{*}{$x_{i}$} & $\begin{array}{c}\text { Tau method } \\
\text { (Shahmorad 2005) }\end{array}$ & $\begin{array}{c}\text { Block-by-Block method } \\
\text { (Hosseini \& Shahmorad, 2002) }\end{array}$ & \multicolumn{2}{|c|}{ Present method } \\
\cline { 2 - 5 } & $N=10$ & $N=10$ & \multicolumn{2}{|c|}{$N=11, M=12$} \\
\cline { 2 - 5 } & $e_{10}\left(x_{i}\right)$ & $e_{10}\left(x_{i}\right)$ & $\left|e_{11}\left(x_{i}\right)\right|$ & $\left|E_{11.12}\left(x_{i}\right)\right|$ \\
\hline 0.0 & 0.000000 & 0.000000 & 2.000000 & 0.000000 \\
\hline 0.2 & $5.721560 \mathrm{e}-12$ & $3.630000 \mathrm{e}-8$ & $2.505511 \mathrm{e}-8$ & $6.86885 \mathrm{e}-9$ \\
\hline 0.4 & $2.384510 \mathrm{e}-8$ & $1.600000 \mathrm{e}-7$ & $2.342665 \mathrm{e}-8$ & $6.05788 \mathrm{e}-9$ \\
\hline 0.6 & $1.049210 \mathrm{e}-4$ & $4.450000 \mathrm{e}-7$ & $2.324619 \mathrm{e}-8$ & $3.58465 \mathrm{e}-9$ \\
\hline 0.8 & $1.049210 \mathrm{e}-4$ & $1.110000 \mathrm{e}-6$ & $2.553052 \mathrm{e}-6$ & $3.33466 \mathrm{e}-7$ \\
\hline 1.0 & $1.615160 \mathrm{e}-3$ & $2.750000 \mathrm{e}-6$ & & \\
\hline
\end{tabular}

Table 3: Comparison of the absolute errors of Eq. (34).

\begin{tabular}{|c|c|c|c|}
\hline \multirow{2}{*}{$x_{i}$} & \multicolumn{3}{|c|}{ Corrected absolute error } \\
\cline { 2 - 4 } & $\left|E_{11.12}\left(x_{i}\right)\right|$ & $\left|E_{11.14}\left(x_{i}\right)\right|$ & $\left|E_{11.17}\left(x_{i}\right)\right|$ \\
\hline 0 & 0 & 0 & 0 \\
\hline 0.2 & $7.34493 \mathrm{e}-9$ & $9.1127 \mathrm{e}-10$ & $2.8085 \mathrm{e}-10$ \\
\hline 0.4 & $6.86885 \mathrm{e}-9$ & $9.9376 \mathrm{e}-10$ & $7.151 \mathrm{e}-11$ \\
\hline 0.6 & $6.05788 \mathrm{e}-9$ & $5.3919 \mathrm{e}-10$ & $3.8486 \mathrm{e}-10$ \\
\hline 0.8 & $3.58465 \mathrm{e}-9$ & $8.8155 \mathrm{e}-10$ & $2.71137 \mathrm{e}-9$ \\
\hline 1.0 & $3.33466 \mathrm{e}-7$ & $1.6776 \mathrm{e}-7$ & $3.98625 \mathrm{e}-9$ \\
\hline
\end{tabular}

Table 4: Numerical results of the error functions of Example 5.3 for $N=11, M=12,14,17$. 


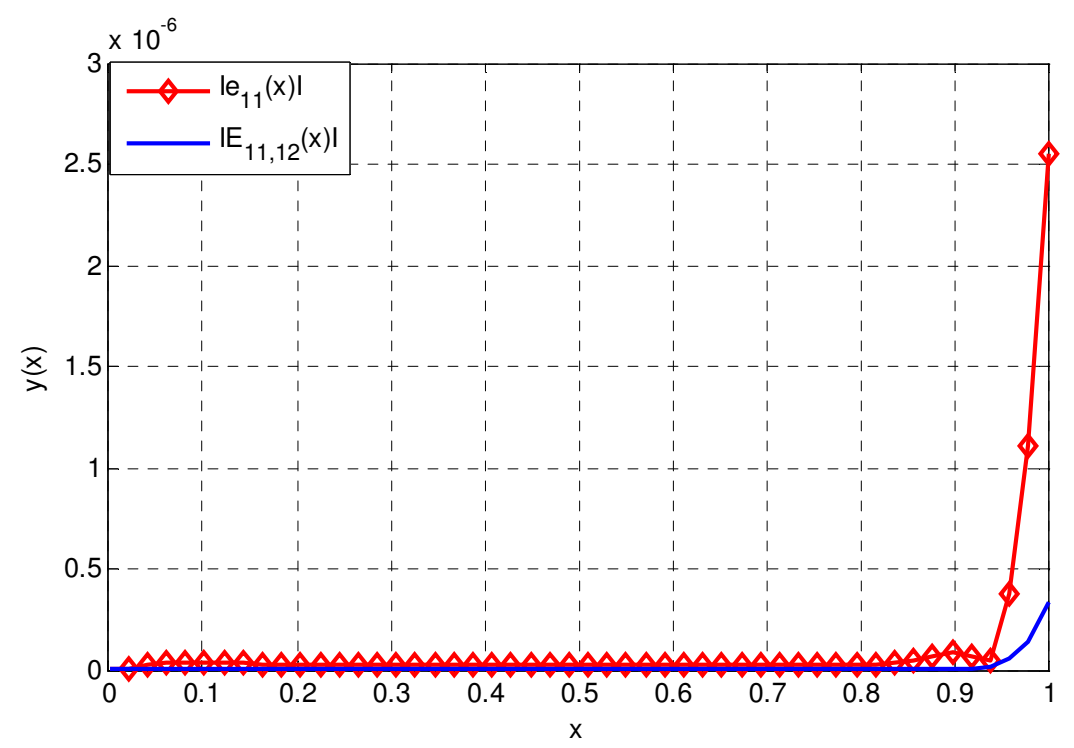

Fig. 2: Comparison of the absolute error with the corrected absolute error for Eq.(34).

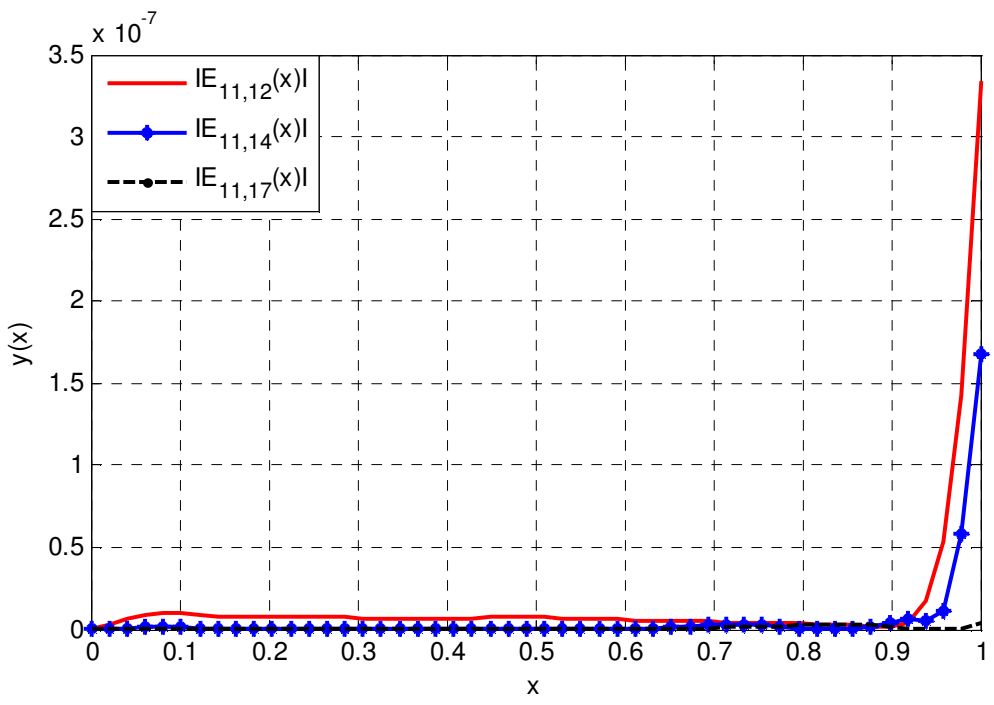

Fig. 3: Comparison of the corrected absolute errors for Eq.(34).

\section{Conclusion}

In this article, we have presented Fibonacci collocation method with a residual error function to solve high order linear Volterra integro-differential equations. From the comparisons of the results obtained by this method is more effective than some other methods. It is observed from discussed examples which have the exact solution, the error estimation algorithm is very effective when the compared absolute errors of these examples. And also, if the exact solution of the problem is 
unknown, the absolute errors can be computed with this error estimation algorithm. One of the considerable advantages of this method is that the approximate solutions are found very easily by using the computer programs. As seen from this study, the Fibonacci polynomial approach is a good approximation for solving these equations.

\section{References}

[1] Brunner H., Davies P.J \& Duncan D.B. 2009. Discontinuous Galerkin approximations for Volterra integral equations of the first kind. IMA J. Numer. Anal. 29: 856-881.

[2] Cao J. \& Wang J. 2004. Delay-dependent robust stability of uncertain nonlinear systems with time delay. Appl. Math. Comput. 154: 289-297.

[3] Çelik İ. 2006. Collocation method and residual correction using Chebyshev series. Applied Mathematics and Computation. 174 (2): 910-920.

[4] Danfu H. \& Xufeng S. 2007. Numerical solution of integro-differential equations by using CAS wavelet operational matrix of integration. Appl. Math. Comput. 194: 460-466.

[5] Elmer C.E. \& Van Vleck E.S. 2002. A variant of Newton's method for solution of traveling wave solutions of bistable differentialdifference equation. J. Dyn. Differ. Equ. 14: 493-517.

[6] Erdem K., Yalcinbas S., Sezer M. 2013. A Bernoulli approach with residual correction for solving mixed linear Fredholmintegrodifferential-difference equations,Journal of Difference Equations and Applications, 19(10): 1619-1631.

[7] Hosseini S.M. \&Shahmorad S. 2002. A matrix formulation of the Tau method and Volterra linear integro-differential equations. Korean J. Comput. Appl. Math. 9 (2): 497-507.

[8] Kadalbajoo, M.K. \& Sharma K.K. 2002. Numerical analysis of boundary-value problems for singularly-perturbed differentialdifference equations with small shifts of mixed type. J. Optim. Theory Appl. 115: 145-163.

[9] Kadalbajoo M.K. \& Sharma K.K. 2004. Numerical analysis of singularly-perturbed delay differential equations with layer behavior. Appl. Math. Comput. 157:11-28.

[10] Kajani M.T., Ghasemi M. \&Babolian E. 2007. Comparison between the homotopy perturbation method and the sine-cosine wavelet method for solving linear integro-differential equations. Comput. Math. Appl. 54: 1162-1168.

[11] Kurt A. 2012. Fibonacci polynomial solutions of linear differential, integral and integro-differential equations, M.Sc. Thesis, Graduate School of Natural and Applied Sciences, Mugla University.

[12] Kurt A., Yalcinbas S. \&Sezer M. 2013. Fibonacci Collocation Method For Solving Linear Differential-Difference Equations. Mathematical and Computational Applications. 18.3: 448-458.

[13] Kurt A., Yalçınbaş S., Sezer M. 2013. Fibonacci Collocation Method for Solving High- Order Linear FredholmIntegro-DifferentialDifference Equations. International Journal of $\quad$ Mathematics and Mathematical Sciences 2013: 1-9.

[14] Makroglou A. 1980. Convergence of a block-by-block method for nonlinear Volterraintegro differential equations, Math. Comput. 35: 783-796.

[15] Maleknejad K. \&ahmoudi Y. 2004. Numerical solution of linear Fredholm integral equation by using hybrid Taylor and blockpulse functions. Appl. Math. Comput. 149: 799-806.

[16] Maleknejad K. \&Mirzaee F. 2006. Numerical solution of integro-differential equations by using rationalized Haar functions method. Kybernetes Int. J. Syst. Math. 35: 1735-1744.

[17] Oliveira FA. 1980. Collocation and residual correction. NumerischeMathematik, 36 (1): 27-31.

[18] Pour-Mahmoud J., Rahimi-Ardabili M.Y \& Shahmorad S. 2005. Numerical solution of the system of Fredholmintegro-differential equations by the Tau method. Appl. Math. Comput. 168: 465-478.

[19] Sezer M. 1994. Taylor polynomial solution of Volterra integral equations. Int. J. Math. Educ. Sci. Technol. $25: 625-633$.

[20] Shahmorad S. 2005. Numerical solution of general form linear Fredholm-Volterraintegro-differential equations by the Tau method with an error estimation. Appl. Math. Comput. 167 (2): 1418-1429.

[21] Sorkun H.H. \& Yalcinbas S. 2010. Approximate solution of linear Volterra integral equation systems with variable coefficients. Applied Mathematical Modelling. 34(11): 3451-3464. 
[22] Wazwaz A.M. 2010. The combined Laplace transform-Adomian decomposition method for handling nonlinear Volterraintegrodifferential equations. Appl. Math. Comput. 216: 1304-1309.

[23] Yalcinbas S. \& Sezer M. 2000. The approximate solution of high-order linear Volterra-Fredholmintegro-differential equations in terms of Taylor polynomials.Appl. Math. Comput. 112(2): 291-308.

[24] Yalcinbas S. 2002. Taylor polynomial solutions of nonlinear Volterra-Fredholm integral equations. Appl. Math. Comput. 127: 195-206.

[25] Yalcinbas S. \& Erdem K. 2010. Approximate solutions of nonlinear Volterra integral equation systems. International Journal of Modern Physics B, 24(32): 6235-6258.

[26] Yalcinbas S., Sahin N. \& Sezer M. 2011. Bessel polynomial solutions of high-order linear Volterraintegro-differential equations. Comput. Math. Appl. 62(4): 1940-1956.

[27] Zhao J \& Corless R.M. 2006. Compact finite difference method for integro-differential equations. Appl. Math. Comput. 177: 271-288. 